\title{
High-Throughput Genotyping of CRISPR/Cas Edited Cells in 96-Well Plates
}

\author{
Lea Nussbaum ${ }^{1,+}$ (iD, Jelena M. Telenius ${ }^{1,2,+}$, Stephanie Hill ${ }^{1}$, Priscila P. Hirschfeld ${ }^{1}$, \\ Maria C. Suciu 1,3 (D), The WIGWAM Consortium ${ }^{4,5}$, Damien J. Downes ${ }^{1, *}$ (i) \\ and Jim R. Hughes $1,2, *$ \\ 1 MRC Molecular Haematology Unit, Weatherall Institute of Molecular Medicine, Oxford OX3 9DU, UK; \\ lea.nussbaum@lincoln.ox.ac.uk (L.N.); jelena.telenius@imm.ox.ac.uk (J.M.T.); \\ stephanie.hill@ndcls.ox.ac.uk (S.H.); priscila.hirschfeld@ndcls.ox.ac.uk (P.P.H.); \\ maria.suciu@genomicsplc.com (M.C.S.) \\ 2 MRC WIMM Center for Computational Biology, Weatherall Institute of Molecular Medicine, \\ Oxford OX3 9DU, UK \\ 3 Genomics plc, Oxford OX1 1JD, UK \\ 4 Weatherall Institute of Molecular Medicine, Oxford OX3 9DU, UK; jim.hughe@imm.ox.ac.uk \\ 5 Wellcome Trust Centre for Human Genetics, Oxford OX3 7BN, UK \\ * Correspondence: damien.downes@ndcls.ox.ac.uk (D.J.D.); jim.hughes@imm.ox.ac.uk (J.R.H.); \\ Tel.: +44-(0)1865-222-374 (D.J.D.); +44-(0)1865-222-113 (J.R.H.) \\ + These authors contributed equally to this work.
}

Received: 25 June 2018; Accepted: 23 July 2018; Published: 1 August 2018

\begin{abstract}
The emergence in recent years of DNA editing technologies-Zinc finger nucleases (ZFNs), transcription activator-like effector (TALE) guided nucleases (TALENs), clustered regularly interspaced short palindromic repeats (CRISPR)/Cas family enzymes, and Base-Editors-have greatly increased our ability to generate hundreds of edited cells carrying an array of alleles, including single-nucleotide substitutions. However, the infrequency of homology-dependent repair (HDR) in generating these substitutions in general requires the screening of large numbers of edited cells to isolate the sequence change of interest. Here we present a high-throughput method for the amplification and barcoding of edited loci in a 96-well plate format. After barcoding, plates are indexed as pools which permits multiplexed sequencing of hundreds of clones simultaneously. This protocol works at high success rate with more than $94 \%$ of clones successfully genotyped following analysis.
\end{abstract}

Keywords: CRISPR/Cas9 genotyping; homology dependent repair; genome editing; genome-wide association study (GWAS) validation

\section{Introduction}

The past two decades have seen the explosion of available genomic editing tools for eukaryotic systems including Zinc finger nucleases (ZFNs) [1], transcription activator-like effector (TALE) guided nucleases (TALENs) [2], CRISPR/Cas nucleases [3-5], and most recently the clustered regularly interspaced short palindromic repeats (CRISPR)/Cas, and Base-Editors [6]. These systems operate via endogenous DNA repair mechanisms to bring about nucleotide changes and have greatly increased the routine throughput of genomic editing experiments. Generally, sequence changes arise during repair of double-strand DNA breaks, with repair primarily carried out by the non-homologous end joining (NHEJ) pathway, which generates small insertions or deletions (indels), and less commonly large deletions $[7,8]$. However, exact base-pair changes can also be generated by the less-frequent homology-dependent repair (HDR) pathway using co-transfected donor or template DNA [9]. 
While many experimental approaches have used pools of CRISPR/Cas guides to edit numerous loci, such as knock-out and drop-out screens [10-13], other experiments require a more tailored approach, using HDR to introducing specific changes within a few loci [9,14]. For example, the exact editing of single nucleotide polymorphisms (SNPs) within primary cells or cell lines is essential to functionally validate causative SNPs identified from genome-wide association studies [15].

Although methods for both editing cells and isolating clones of edited cells have become much more accessible, techniques for high-throughput screening of large numbers of clones are still required. These techniques are particularly important for projects where exact nucleotide changes are required as HDR occurs much less frequently than NHEJ, requiring larger numbers of clones to be screened to isolate correctly repaired loci $[7,12,16]$. While it is often possible to identify sequence changes via traditional Sanger sequencing these signals become confused by heterozygotes with small indels and are hard to incorporate into high-throughput pipelines; this is especially complex in polyploid cells with more than two alleles.

Conventional screening approaches use Sanger sequencing [17], which is low-throughput and poorly resolves heterozygotes. Alternatively, polymerase chain reaction (PCR)-capillary gel electrophoresis [18] and high-resolution melting-curve analysis [19] are high-throughput methods, but provide no sequence information. In contrast, next-generation sequencing generates signal from a single allele and makes signal deconvolution unnecessary for heterozygotes, while at the same time, allows high-throughput handling [20]. We have, therefore, developed a pipeline for the high-throughput genotyping of targeted loci in edited and single-cell sorted clones. Our method uses three rounds of amplification: first to isolate the locus of interest, second to barcode the well into which the clone was sorted, and third to index the specific plate. This method allows for several hundred clones to be genotyped simultaneously in a single sequencing run and also permits the multiplexed screening of editing at multiple loci. Importantly the protocol provides exact allelic sequence for resolution of complex alleles in diploid or polyploid cells and provides sequence files that can be analyzed with provided plateScreen96 scripts, or input into other analysis tools (e.g., OutKnocker [21]). This protocol will be highly useful for studies where isolation of clones with exact nucleotide changes is necessitated; and may be easily incorporated into an automated robotics system for even higher throughput applications.

\section{Experimental Design}

This protocol describes the genotyping of one or more loci of interest using next-generation sequencing. The protocol requires cells to have previously been edited, single-cell sorted and clonally expanded following a cell-type specific protocol (e.g., HEK-293 [22] or HUDEP-2 [23]). Expanded cells are split with one plate stored frozen and the other used for genotyping. As a control for this protocol it is appropriate to include one sample well from unedited cells.

Genotyping (Figure 1) begins with the amplification of one or more loci of interest using specific primers which contain a linker sequence. The linker sequence is then used as a primer for a second round of amplification in which, maximally distinguishable combinations of barcodes are incorporated in a well-by-well basis. Each plate of PCR products is then pooled together for the addition of next-generation sequencing adaptors and indices, which allows multiplexing of numerous plates and the simultaneous sequencing of hundreds, or even thousands, of clones. Fastq files from sequencing are then analyzed using the plateScreen 96 code $[24,25]$ which reconstructs the original DNA fragments using overlapping forward and reverse reads (flashing). These flashed sequences are then mapped back to an appropriate genome build, and each unique allele along with how many times it was sequenced is reported. The output of plateScreen 96 is an easily readable pdf report summarizing the genotypes of the genome edits in each well. Once the desired clones are identified these can be recovered from freezer storage and expanded. 

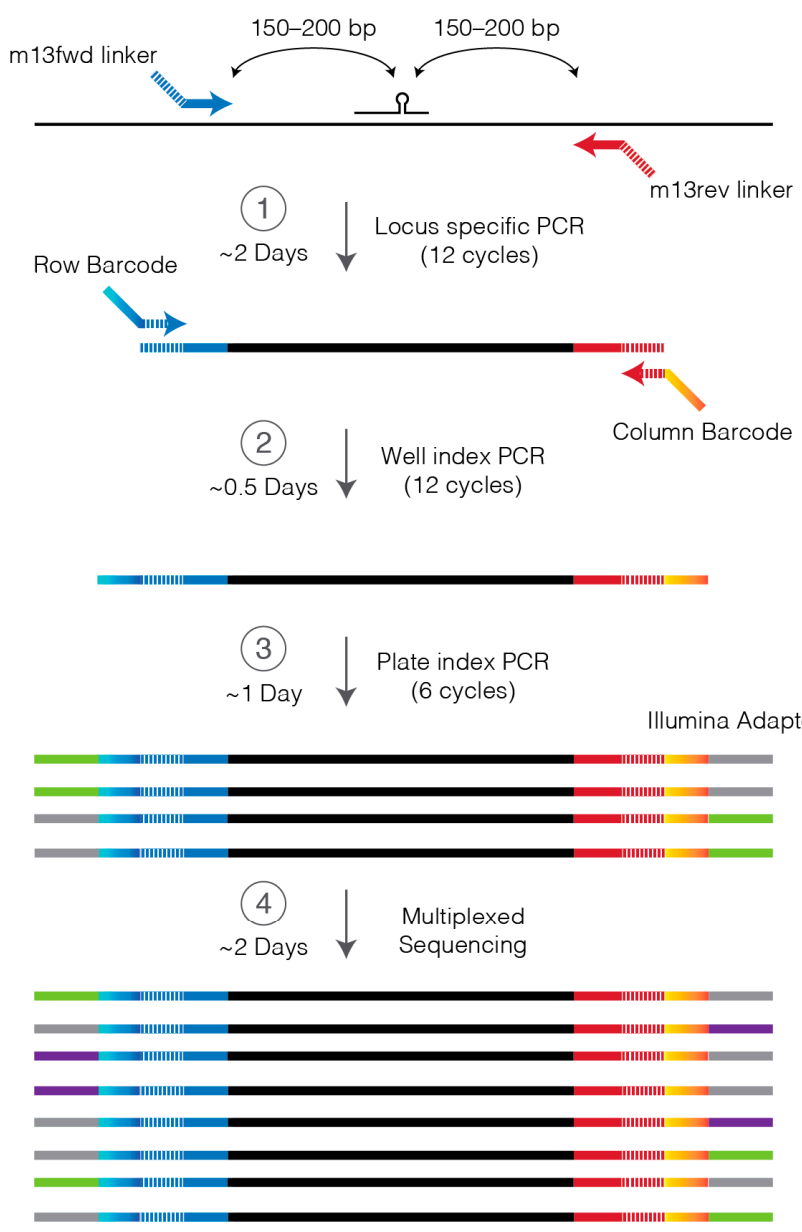

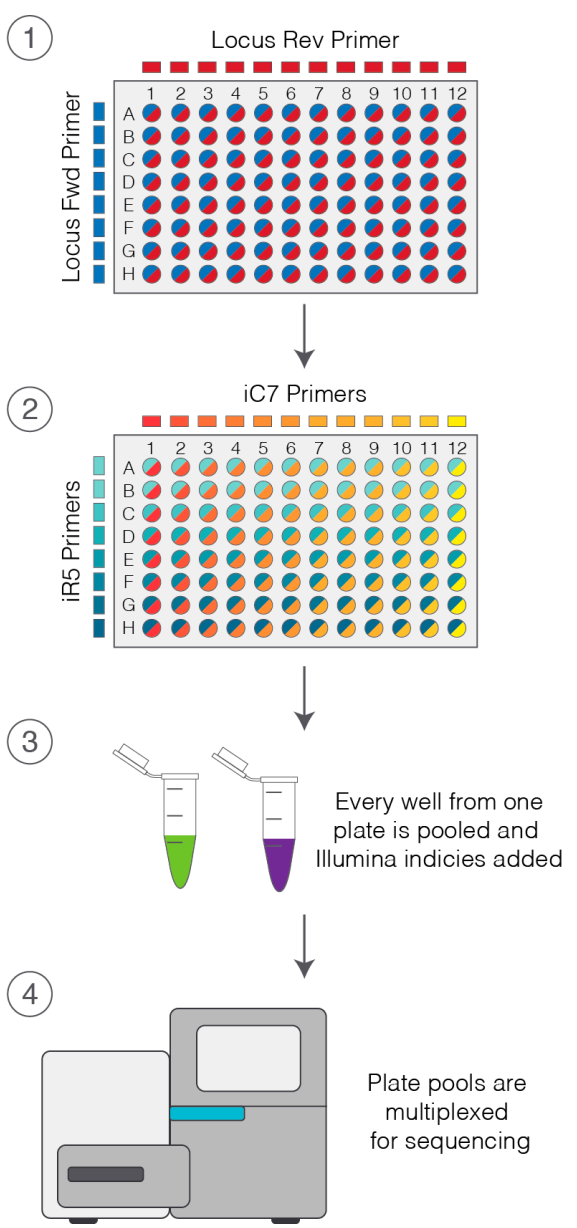

Figure 1. Workflow for 96-well plate amplification of edited loci from lysed cells (1), indexing of each well with a unique barcode primer pair (2), pooling of up to 96 wells from one plate for addition of Illumina adaptors and indices (3), followed by next-generation sequencing of pooled libraries from multiple plates (4). PCR: Polymerase chain reaction; fwd; forward; rev: reverse.

\subsection{Reagents and Materials}

- $\quad$ Fetal bovine serum, FBS (Thermo Fisher, Paisley, UK; Cat. no.: 10270-106)

- $\quad$ Dimethyl sulphoxide, DMSO (VWR, Lutterworth, UK; Cat. no.: 23500.260)

- $\quad$ 96-well V-bottomed plates (Sigma-Aldrich, Dorset, UK; Cat. no.: CLS3894)

- $\quad$ Parafilm (VWR, Lutterworth, UK; Cat. no.: PM-996)

- 96-well PCR plate (Thermo Fisher; Cat. no.: AB1400L)

- $\quad$ Tris, 1 M, pH 8.0 (Thermo Fisher; Cat. no.: AM9855G)

- $\quad$ Ethylenediaminetetraacetic acid (EDTA), 0.5 mM, pH 8.0 (Thermo Fisher; Cat. no.: 15575-038)

- Tween 20,50\% (Thermo Fisher; Cat. no.: 003005)

- PCR grade water (Thermo Fisher; Cat. no.: AM9932)

- DNA low bind tubes (Eppendorf, Arlington, UK; Cat. no.: Z666548)

- Proteinase K (Thermo Fisher; Cat. no.: EO0491)

- Platinum PCR master mix (Thermo Fisher; Cat. no.: 12532016)

- Locus specific primers, $10 \mu \mathrm{M}$ mix, see Section 2.3 (Sigma, St Louis, MO, USA or Integrated DNA Technologies (IDT), Skokie, Illinois, USA)

- $\quad$ Agarose (Roche, Burgess Hill, UK; Cat. no.: 11388983001)

- $10 \times$ Tris acetate-EDTA, TAE (Sigma-Aldrich; Cat. no.: 11666690001) 
- 100 bp ladder (New England Biolabs, Hitchin, UK; Cat. no.: N0551G)

- Exonuclease I, Escherichia coli (New England Biolabs; Cat. no: M0293L)

- $\quad$ Shrimp alkaline phospatase, rSAP (New England Biolabs, Hitchin, UK; Cat. no.: M0371L)

- Custom iR5 and iC7 barcoded primers, Table 1 (Sigma or IDT)

- Agencourt Ampure XP SPRI Beads (Beckman Coulter, High Wycombe, UK; Cat. no.: A63881)

- $100 \%$ Ethanol (VWR, Lutterworth, UK; Cat. no.: 20821.330)

- Qubit BR DNA assay kit (Thermo Fisher; Cat. no.: Q32850)

- $\quad$ NEB Ultra II (New England Biolabs; Cat. no.: 7645S/L)

- $\quad$ PCR Tube (Appleton Woods, Birmingham, UK; Cat. no.: TA571)

- NEB Next Multiplex Oligos for Illumina primer set 1 (New England Biolabs; Cat. no.: E7500S/L)

- NEB Next Multiplex Oligos for Illumina primer set 2 (New England Biolabs; Cat. no.: E7335S/L)

- Herculase II Fusion polymerase kit (Agilent, Cheadle, UK; Cat. no.: 600677)

- Tris-EDTA, TE (Sigma-Aldrich; Cat. no.: 99302)

- D1000 reagents (Agilent; Cat. no.: 50675583)

- D1000 loading tips (Agilent; Cat. no.: 50675153)

- D1000 screen tape (Agilent; Cat. no.: 50675582)

- KAPA Library Quantification Complete Kit (Roche; Cat. no.: KK4824)

- $\quad$ MiSeq Reagent Nano Kit, v2 500-Cycles (Illumina, Cambridge, UK; Cat. no.: MS-103-1003)

- PhiX Control v3 (Illumina; Cat. no.: FC-110-3001)

\subsection{Equipment}

- 8- or 12-channel pipette (Labgene Scientific, Châtel-Saint-Denis, Switzerland: Cat. no.: 5121, 5125)

- Centrifuge with buckets for plates (Eppendorf; Cat. no.: 5810R)

- Thermocycler with 96-well plate capacity (Bio-Rad, Watford, UK; Cat. no.: T100)

- Electrophoresis gel tank and power pack

- Magnetic rack (DynaMag-2; Thermo Fisher; Cat. no.: 13221)

- $\quad$ Minifuge (Starlab, Milton Keynes, UK; Cat. no.: N2631-0007)

- Qubit fluorometer (Thermo Fisher; Cat. no.: Q33226)

- $\quad$ Agilent 2200 TapeStation (Agilent; Cat. no.: G2964AA)

- Real-time quantitative polymerase chain reaction (qPCR) thermocycler (Thermo Fisher StepOnePlus; Thermo Fisher; Cat. no.: 4376598)

- $\quad$ MiSeq (Illumina; Cat. no.: SY-410-1003)

- $\quad$ Microcentrifuge (Eppendorf; Cat. no.: 5424R)

\subsection{Custom Locus Primers}

The first PCR step of this protocol requires amplification of the edited locus using custom primers (Supplementary Materials Table S1). Primers are designed using standard tools such as IDT PrimerQuest [26] and should be positioned between 150-200 bp from the editing site to generate a 300-400 bp PCR product. When screening for HDR events is essential primers do not overlap any donor sequence (such as single stranded oligodeoxynucleotides, ssODNs) to avoid amplification from incorrect insertions. Additionally, distant primers minimize the likelihood of false-positive homozygotes generated by a large deletion on one allele removing a primer binding site. False homozygotes can be filtered either with a heterozygous SNP within the PCR product, or by screening for heterozygosity with a larger PCR product ( $>5 \mathrm{~kb}$ in size) to identify large deletions. However, placement of primers should be such that the final PCR product is no greater than $\sim 400 \mathrm{bp}$, as this enables sequence coverage across the entire amplicon and generates overlapping reads to reconstruct the original PCR product, which is essential for complete analysis. Up to five primer pairs are designed and tested in silico with BLAT [27] to ensure site locus specificity. A modified m13fwd 
(5'-GTAAACGACGGCCAGT-3') and m13rev (5'-AGCGGATAACAATTTCACACAGGA-3') sequence are then added to the $5^{\prime}$ ends of the forward and reverse primers respectively. The $\mathrm{m} 13$ sequences serve dual purposes, acting as adaptors for barcode primer binding and allowing for traditional Sanger sequencing if necessary.

As the addition of the m13 linkers may alter the binding specificity of the primer, at least two primer pairs are ordered and tested on genomic DNA (Figure 2). The PCR products from these primers can be sequenced to confirm specificity if necessary. Primer-pair cocktails are made by mixing equal volumes of $20 \mu \mathrm{M}$ forward and reverse primer dilutions to generate a working stock containing each primer at $10 \mu \mathrm{M}$.

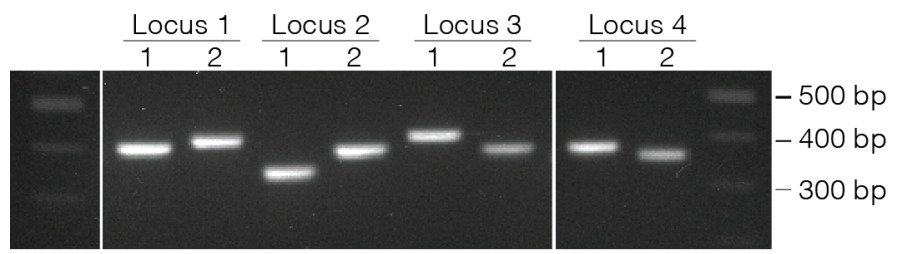

Figure 2. Two pairs of primers are validated for each edited locus with genomic DNA from the cells to be edited and running on a $2 \%$ agarose gel.

\subsection{Primers to Barcode Individual Wells}

Locus specific PCR products are barcoded with one of eight forward primers (iR5) and one of twelve reverse primers (iC7) in all 96 permutations to uniquely identify each well (Figure 2). These primers contain a $3 \mathrm{bp}$ spacer (GAT), and $8 \mathrm{bp}$ barcode, and a modified m13fwd or m13rev sequence (Table 1) to prime from the locus specific PCR product. Primers should be made up to a working stock of $10 \mu \mathrm{M}$.

Table 1. Oligonucleotides for barcoding 96-wells.

\begin{tabular}{ccc}
\hline ID & Barcode & Sequence \\
\hline & & iR5 - Row Barcode Primers (Forward) \\
\hline iR5_A & TATAGCCT & GATTATAGCCTGTAAAACGACGGCCAGT \\
iR5_B & ATAGAGGC & GATATAGAGGCGTAAAACGACGGCCAGT \\
iR5_C & CCTATCCT & GATCCTATCCTGTAAAACGACGGCCAGT \\
iR5_D & GGCTCTGA & GATGGCTCTGAGTAAAACGACGGCCAGT \\
iR5_E & AGGCGAAG & GATAGGCGAAGGTAAAACGACGGCCAGT \\
iR5_F & TAATCTTA & GATTAATCTTAGTAAAACGACGGCCAGT \\
iR5_G & CAGGACGT & GATCAGGACGTGTAAAACGACGGCCAGT \\
iR5_H & GTACTGAC & GATGTACTGACGTAAAACGACGGCCAGT \\
\hline & & iC7-Column Barcode Primers (Reverse) \\
\hline iC701 & ATTACTCG & GATATTACTCGAGCGGATAACAATTTCACACAGGA \\
iC702 & TCCGGAGA & GATCCGGAGAAGCGGATAACAATTTCACACAGGA \\
iC703 & CGCTCATT & GATCGCTCATTAGCGGATAACAATTTCACACAGGA \\
iC704 & GAGATTCC & GATGAGATTCCAGCGGATAACAATTTCACACAGGA \\
iC705 & ATTCAGAA & GATATTCAGAAAGCGGATAACAATTCACACAGGA \\
iC706 & GAATTCGT & GATGAATTCGTAGCGGATAACAATTTCACACAGGA \\
iC707 & CTGAAGCT & GATCTGAAGCTAGCGGATAACAATTTCACACAGGA \\
iC708 & TAATGCGC & GATTAATGCGCAGCGGATAACAATTTCACACAGGA \\
iC709 & CGGCTATG & GATCGGCTATGAGCGGATAACAATTTCACACAGGA \\
iC710 & TCCGCGAA & GATCCGCGAAAGCGGATAACAATTTCACACAGGA \\
iC711 & TCTCGCGC & GATTCTCGCGCAGCGGATAACAATTTCACACAGGA \\
iC712 & AGCGATAG & GATAGCGATAGAGCGGATAACAATTTCACACAGGA \\
\hline
\end{tabular}




\subsection{Analysis Software}

The custom plateScreen96 scripts are available on GitHub [24], with additional test files, manual and system requirements also available online [25].

\section{Procedure}

Here we provide instructions for splitting non-adherent cells into two plates, one for freezing and storage (stock plate) and the other for lysis (genotype plate), amplification and indexing producing a next-generation sequence ready library (Figure 1). Cells should already have been edited, single-cell fluorescence-activated cell sorting (FACS) separated and expanded in a method appropriate for the cell type (e.g., HEK-293 [22]; HUDEP-2 [23]). Where single-cell FACS is not available, limiting dilution may be used but care should be made to ensure each well is occupied by a unique clone.

\subsection{Clonal Expansion. Time for Completion: 2-3 Weeks}

1. Using the appropriate growth media, clonally expand single-cell sorted colonies, splitting them as necessary until they occupy two to four wells of a 96-well plate at a high level of confluence $(80-90 \%)$.

\subsection{Splitting and Freezing Cells. Time for Completion: $3 \mathrm{~h}$}

2. Reduce media volume to $<100 \mu \mathrm{L}$ per well and mix by pipetting.

3. Prepare two new 96-well V-bottomed plates (one for stock storage and one for genotyping) by combining two wells of highly confluent cells. Note: If cells have only grown to occupy two wells of a flat-bottomed 96-well plate, transfer a single well to each V-bottomed plate.

A CRITICAL STEP Clones should occupy identical wells in both the stock and genotyping plates.

4. Set aside the genotyping plate and pellet the cells in the stock plate by centrifugation $(250 \times g$, $5 \mathrm{~min}$, room temperature).

5. During this spin step, prepare $5 \mathrm{~mL}$ of sterile freezing media ( $90 \% \mathrm{FBS}, 10 \% \mathrm{DMSO} v / v)$ per stock plate.

6. After centrifugation use an 8- or 12-channel pipette to carefully remove supernatant from the pelleted cells in the stock plate.

A CRITICAL STEP Take care not to dislodge and discard cells by disturbing the pellet.

7. Quickly and carefully resuspend cells in $50 \mu \mathrm{L}$ of freezing buffer.

8. Wrap the stock plate in parafilm, place in polystyrene box or freezing box and store at $-80^{\circ} \mathrm{C}$.

\subsection{Cell Lysis. Time for Completion: Overnight (18 h)}

9. $\quad$ Prepare $3 \mathrm{~mL}$ lysis buffer per full plate by adding proteinase $\mathrm{K}$ (see Section 5 ).

10. Pellet cells in the genotyping plate from step 4 in Section $3.2(250 \times g, 5 \mathrm{~min}$, room temperature).

11. Using an 8- or 12-channel pipette remove supernatant and resuspend in $30 \mu \mathrm{L}$ lysis buffer.

12. Seal plate and incubate at $37^{\circ} \mathrm{C}$ overnight.

13. On the following day, heat to $95^{\circ} \mathrm{C}$ for $10 \mathrm{~min}$ in thermocycler to deactivate proteinase $\mathrm{K}$.

14. Cool to $4{ }^{\circ} \mathrm{C}$

(1) PAUSE STEP Lysed cells can be stored at $-20{ }^{\circ} \mathrm{C}$ for up to one month or at $-80^{\circ} \mathrm{C}$ for one year.

\subsection{Locus Amplification and PCR Product Clean-Up. Time for Completion: $4 \mathrm{~h}$}

15. On ice, prepare $1150 \mu \mathrm{L}$ of Locus PCR master mix per lysed plate by combining $1125 \mu \mathrm{L}$ Platinum master mix and $25 \mu \mathrm{L}$ of $10 \mu \mathrm{M}$ locus primer mix (Section 2.3). 
16. Aliquot $11.5 \mu \mathrm{L}$ of Locus PCR master mix into each well of a 96-well PCR plate on ice.

17. On ice, add $1 \mu \mathrm{L}$ of cell lysate to the corresponding well of the PCR plate and mix by pipetting.

18. Place in thermocycler and amplify using the Platinum PCR cycling settings in Table 2.

Table 2. Platinum polymerase chain reaction (PCR) Amplification.

\begin{tabular}{ccc}
\hline Step & Temp. & Time \\
\hline Step 1 & $94{ }^{\circ} \mathrm{C}$ & 2 min \\
\hline Step 2 & $94{ }^{\circ} \mathrm{C}$ & $30 \mathrm{~s}$ \\
\hline Step 3 & $62{ }^{\circ} \mathrm{C}$ & $30 \mathrm{~s}$ \\
\hline Step 4 & $68^{\circ} \mathrm{C}$ & $1 \mathrm{~min}$ \\
\hline Repeat steps $2-4$ for a total of 12 cycles \\
\hline Step 5 & $72{ }^{\circ} \mathrm{C}$ & 10 min \\
\hline Step 6 & $4{ }^{\circ} \mathrm{C}$ & $\infty$ \\
\hline
\end{tabular}

19. Transfer $1 \mu \mathrm{L}$ of PCR products to the corresponding well of a new 96-well PCR plate.

20. Prepare $220 \mu \mathrm{L}$ PCR clean-up master mix per plate by combining $16.5 \mu \mathrm{L}$ exonuclease I, $16.5 \mu \mathrm{L}$ shrimp alkaline phosphatase (SAP) and $187 \mu \mathrm{L}$ PCR grade water.

21. Add $2 \mu \mathrm{L}$ of PCR clean-up master mix to each well, mix by pipetting.

22. Incubate in a thermocycler at $37^{\circ} \mathrm{C}$ for $30 \mathrm{~min}, 85^{\circ} \mathrm{C}$ for $15 \mathrm{~min}$, and then cool to $4{ }^{\circ} \mathrm{C}$.

(1) PAUSE STEP After stopping the reaction, the mix can be stored at $4{ }^{\circ} \mathrm{C}$ overnight.

3.5. Well Barcoding and PCR Product Clean-Up. Time for Completion: $3 \mathrm{~h}$

23. To each well of cleaned-up PCR product add $11.5 \mu \mathrm{L}$ Platinum master mix (perform on ice).

24. Prepare a stock 96-well plate of barcoding primers by combining all unique pairs of row and column primers at $5 \mu \mathrm{M}$ each by adding equal volumes $(2-5 \mu \mathrm{L})$ of each $\mathrm{iC} 7$ and iR5 primer at $10 \mu \mathrm{M}$. This primer plate may be stored at $-20^{\circ} \mathrm{C}$ and used multiple times.

25. Using the barcoding primers prepared in step 24 and a multichannel pipette add $0.5 \mu \mathrm{L}$ of primers to the appropriate wells of the genotyping plate.

26. Place in a thermocycler and amplify using the Platinum PCR cycling settings in Table 2.

27. During amplification bring an aliquot of $875 \mu \mathrm{L}$ Ampure XP beads to room temp in an Eppendorf tube.

28. Pool $5 \mu \mathrm{L}$ from each well of a single plate into a single $1.5 \mathrm{~mL}$ DNA low bind tube ( $480 \mu \mathrm{L}$ in total). Store excess PCR reaction at $-20^{\circ} \mathrm{C}$.

29. Add $864 \mu \mathrm{L}$ Ampure XP beads (1.8 volumes) to the pooled PCR products. Mix by pipetting.

30. Incubate at room temp for $5 \mathrm{~min}$.

31. During incubation prepare $80 \%$ ethanol ( $1200 \mu \mathrm{L} 100 \%$ ethanol, $300 \mu \mathrm{L}$ PCR grade water).

32. Place on magnetic stand. After the liquid has cleared $\sim 5 \mathrm{~min}$ ), remove and discard the liquid without disturbing the beads.

33. Add $700 \mu \mathrm{L}$ of fresh $80 \%$ ethanol again without disturbing beads. Incubate $30 \mathrm{~s}$.

34. Remove the ethanol and the repeat wash with another $700 \mu \mathrm{L}$ of fresh $80 \%$ ethanol.

35. Discard the ethanol, spin briefly on a bench-top centrifuge and replace on magnetic stand.

36. Discard the residual ethanol and allow to air dry until beads appear matte in appearance $\sim 5 \mathrm{~min}$ ).

A CRITICAL STEP Do not over dry the beads as this will reduce yield. Beads should appear like damp mud, neither glossy wet nor dry. Cracks in the bead pellet are indicative of over-drying. 
37. Remove tube from magnet and resuspend beads in $55 \mu \mathrm{L}$ of PCR grade water by pipetting 10 times.

38. Incubate at room temp for $2 \mathrm{~min}$.

39. Replace on magnetic stand.

40. Once clear ( 4 $\mathrm{min})$ recover $53 \mu \mathrm{L}$ of eluted PCR product and transfer to a new $1.5 \mathrm{~mL}$ DNA low-bind tube.

41. Use $2 \mu \mathrm{L}$ of eluted PCR to quantify the DNA concentration using a Qubit BR DNA kit.

42. Use $1 \mu \mathrm{L}$ of eluted PCR to evaluate the product size on a D1000 TapeStation gel.

(1) PAUSE STEP After purification the products can be stored at $4{ }^{\circ} \mathrm{C}$ overnight or at $-20{ }^{\circ} \mathrm{C}$ for several months.

\subsection{Addition of Illumina Adaptors. Time for Completion: $3 \mathrm{~h}$}

These steps follow the NEB Ultra II protocol with minor modifications.

43. Combine $50 \mu \mathrm{L}$ of DNA (0.5-2 $\mu \mathrm{g}), 7 \mu \mathrm{L} 10 \times$ End Prep Buffer and $3 \mu \mathrm{L}$ End Prep Enzyme. Mix by pipetting and incubate at $20^{\circ} \mathrm{C}$ for $30 \mathrm{~min}$ in a thermocycler (lid open or un-heated).

44. Increase temperature to $65^{\circ} \mathrm{C}$ and incubate for $30 \mathrm{~min}$ (lid heated to $75^{\circ} \mathrm{C}$ ).

45. Add $30 \mu \mathrm{L}$ Ultra II Ligation Master Mix, $2.5 \mu \mathrm{L}$ NEBNext Adaptor, $1 \mu \mathrm{L}$ Ligation enhancer. Mix by pipetting and incubate at $20^{\circ} \mathrm{C}$ for $15 \mathrm{~min}$.

46. Add $3 \mu \mathrm{L}$ USER ${ }^{\mathrm{TM}}$ enzyme. Mix by pipetting and incubate at $37^{\circ} \mathrm{C}$ for $15 \mathrm{~min}$.

47. During this USER ${ }^{\mathrm{TM}}$ enzyme reaction warm $90 \mu \mathrm{L}$ of Ampure XP beads to room temp.

48. Add $87 \mu \mathrm{L}$ Ampure $\mathrm{XP}$ beads $(0.9 \times)$ and mix by pipetting. Incubate at room temp for $5 \mathrm{~min}$.

49. During incubation prepare $80 \%$ ethanol ( $800 \mu \mathrm{L} \mathrm{100 \%}$ ethanol, $200 \mu \mathrm{L}$ PCR grade water).

50. Place on magnetic stand. Without disturbing beads, discard liquid when clear ( $\sim 5 \mathrm{~min})$.

51. Add $500 \mu \mathrm{L}$ of fresh $80 \%$ ethanol without disturbing beads. Incubate $30 \mathrm{~s}$.

52. Remove ethanol and repeat wash with another $500 \mu \mathrm{L}$ of fresh $80 \%$ ethanol.

53. Discard ethanol, spin briefly on a bench-top centrifuge and replace on magnetic stand.

54. Discard residual ethanol and allow to air dry until beads are matte in appearance ( $\sim \mathrm{min})$.

A CRITICAL STEP Do not over dry the beads as this will reduce yield. Beads should appear like damp mud, neither glossy wet nor dry. Cracks in the bead pellet are indicative of over-drying.

55. Remove tube from magnet and resuspend beads in $30.5 \mu \mathrm{L}$ of PCR grade water by pipetting 10 times.

56. Incubate at room temperature for $2 \mathrm{~min}$.

57. Replace on magnetic stand.

58. Once clear $\sim 4 \mathrm{~min}$ ) recover $28.5 \mu \mathrm{L}$ of eluted PCR product and transfer to a new PCR tube.

\subsection{Indexing for Multiplexing of Multiple Plates. Time for Completion: $2 \mathrm{~h}$}

59. To the adaptor ligated library add:

- $\quad 5 \mu \mathrm{L}$ NEB Universal Primer

- $\quad 5 \mu \mathrm{L}$ NEB Index Primer

- $\quad 10 \mu \mathrm{L}$ Herculase II $5 \times$ Buffer

- $\quad 0.5 \mu \mathrm{L}$ deoxynucleotide triphosphate (dNTP)

- $\quad 1 \mu \mathrm{L}$ Herculase II Polymerase

60. Mix by pipetting and amplify using the Herculase PCR cycling settings in Table 3 . 
Table 3. Herculase PCR Amplification.

\begin{tabular}{ccc}
\hline Step & Temp. & Time \\
\hline Step 1 & $98^{\circ} \mathrm{C}$ & $30 \mathrm{~s}$ \\
\hline Step 2 & $98^{\circ} \mathrm{C}$ & $10 \mathrm{~s}$ \\
\hline Step 3 & $65^{\circ} \mathrm{C}$ & $30 \mathrm{~s}$ \\
\hline Step 4 & $72{ }^{\circ} \mathrm{C}$ & $30 \mathrm{~s}$ \\
\hline Repeat steps $2-4$ for a total of 6 cycles \\
\hline Step 5 & $72{ }^{\circ} \mathrm{C}$ & 5 min \\
\hline Step 6 & $4{ }^{\circ} \mathrm{C}$ & $\infty$ \\
\hline
\end{tabular}

61. During the PCR warm $40 \mu \mathrm{L}$ of Ampure $\mathrm{XP}$ beads to room temp.

62. Add $40 \mu \mathrm{L}$ Ampure $\mathrm{XP}$ beads $(0.8 \times)$ to the $\mathrm{PCR}$ and mix by pipetting. Incubate at room temperature for $5 \mathrm{~min}$.

63. During incubation prepare $80 \%$ ethanol ( $800 \mu \mathrm{L} \mathrm{100 \%}$ ethanol, $200 \mu \mathrm{L}$ PCR grade water).

64. Place on magnetic stand. Without disturbing beads, discard liquid when clear $\sim 5 \mathrm{~min}$ ).

65. Add $500 \mu \mathrm{L}$ of fresh $80 \%$ ethanol without disturbing beads. Incubate $30 \mathrm{~s}$.

66. Remove ethanol and repeat wash with another $500 \mu \mathrm{L}$ of fresh $80 \%$ ethanol.

67. Discard ethanol, spin briefly on a bench-top centrifuge and replace on magnetic stand.

68. Discard residual ethanol and allow to air dry until beads are matte in appearance ( $\sim \mathrm{min})$.

A CRITICAL STEP Do not over dry the beads as this will reduce yield. Beads should appear like damp mud, neither glossy wet nor dry. Cracks in the bead pellet are indicative of over-drying.

69. Remove tube from magnet and resuspend beads in $30 \mu \mathrm{L}$ of $0.1 \times$ Tris-EDTA (TE) by pipetting 10 times.

70. Incubate at room temperature for $2 \mathrm{~min}$.

71. Replace on magnetic stand. Once clear ( $\sim 4 \mathrm{~min})$ recover $28 \mu \mathrm{L}$ of eluted PCR product and transfer to a new DNA low bind tube.

72. Confirm PCR product size using either a $2 \%$ agarose gel or D1000 TapeStation.

(1) PAUSE STEP After this step the indexed library can be stored at $4{ }^{\circ} \mathrm{C}$ overnight or at $-20^{\circ} \mathrm{C}$ for several months.

\subsection{Quantification and Sequencing. Time for Completion: $28 \mathrm{~h}$}

73. Make a 1:10,000 and 1:20,000 dilution of indexed PCR products and quantify by real-time qPCR using the KAPA Illumina quantification kit.

74. If sequencing in-house prepare $8 \mathrm{nM}$ dilutions of each PCR plate pool and combine differently indexed plates in equal volumes.

75. If sequencing in a core facility prepare DNA pools to their specifications.

76. Sequence on a MiSeq using a 500-cycle Nano kit (250 bp reads, paired end) with $10 \%$ PhiX.

A CRITICAL STEP Locus-specific PCR products will have near identical sequences, PhiX is essential to avoid MiSeq run failure from low complexity. If sequencing a single locus specific PCR product, increase PhiX to $30 \%$.

\subsection{Data Analysis. Time for Completion: 1-3 Days}

All scripts as well as sample files and fastq files (analyzed below) are available via GitHub as a user tutorial. 
77. Download fastq files from either BaseSpace or core facility provider.

78. In a single UNIX directory prepare the following metadata plain text files:

1. PIPE_fastqPasths.txt: Containing names and paths to fastq files.

2. PIPE_spacerBarcodePrimer_FWD.txt: Containing forward primer sequences.

3. PIPE_spacerBarcodePrimer_REV.txt: Containing reverse primer sequences.

4. PIPE_targetLocus_xxX.bed: Locus PCR amplification coordinates and additional region(s) of interest highlighted (Note: species of interest replaces $\mathrm{xxX}$ in the file name, e.g., PIPE_targetLocus_hg19.bed)

A CRITICAL STEP Plain text files are essential as rich text files will not be read correctly and cause analysis failure.

79. Run analysis with the following minimal command (additional parameters are available): $>$./plateScreen96.sh-g hg19.

80. Analyze pipe output and select clones to recover (see Expected Results).

\subsection{Recovery of Clones of Interest. Time for Completion: $2 \mathrm{~h}$}

81. Prepare one $1.5 \mathrm{~mL}$ microcentrifuge tube per clone by adding $1 \mathrm{~mL}$ sterile phosphate-buffered saline (PBS).

82. Recover plate from $-80^{\circ} \mathrm{C}$ storage and remove parafilm.

83. Working quickly, add $150 \mu \mathrm{L}$ of sterile PBS to wells containing clones to be recovered.

84. As each clone thaws, pipette mix to quickly combine with PBS, transfer melted clones to microcentrifuge tubes containing PBS.

85. Centrifuge to pellet cells $(250 \times g, 5 \mathrm{~min})$.

86. Discard supernatant and resuspend in appropriate amount of growth media and plate for expansion.

\section{Expected Results}

During amplification and indexing of the edited locus, quality-control checks that are performed ensure libraries are successfully prepared for sequencing (Figure 3). Analysis of sequenced clones using plateScreen96 custom scripts will provide numerous metadata files including sequence quality, metrics of flashing, and importantly a PDF/PNG report (Figure 4). The report includes important metadata from the analysis, including barcode and adaptor sequences, reference genome coordinates and sequence, optional cell line sequence, and finally reads from each clone sorted by well and reporting PCR number. The aligned reads allow manual inspection to determining editing outcome and identify clones of interest. Although performing at a high success rate, not all wells generate sufficient sequence (threshold 10 reads), to be reported (Table 4). The success of sequencing is highly dependent on the number of cells used at the initial lysis step, with two near confluent wells from a 96-well plate providing the optimal results. 


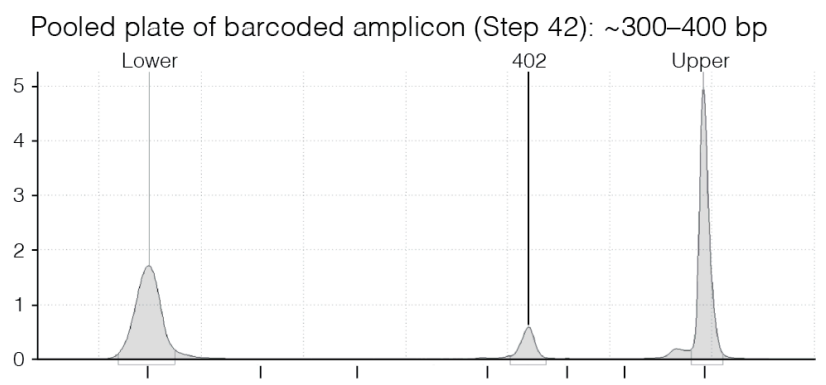

Post Adaptor Ligation (Step 58)
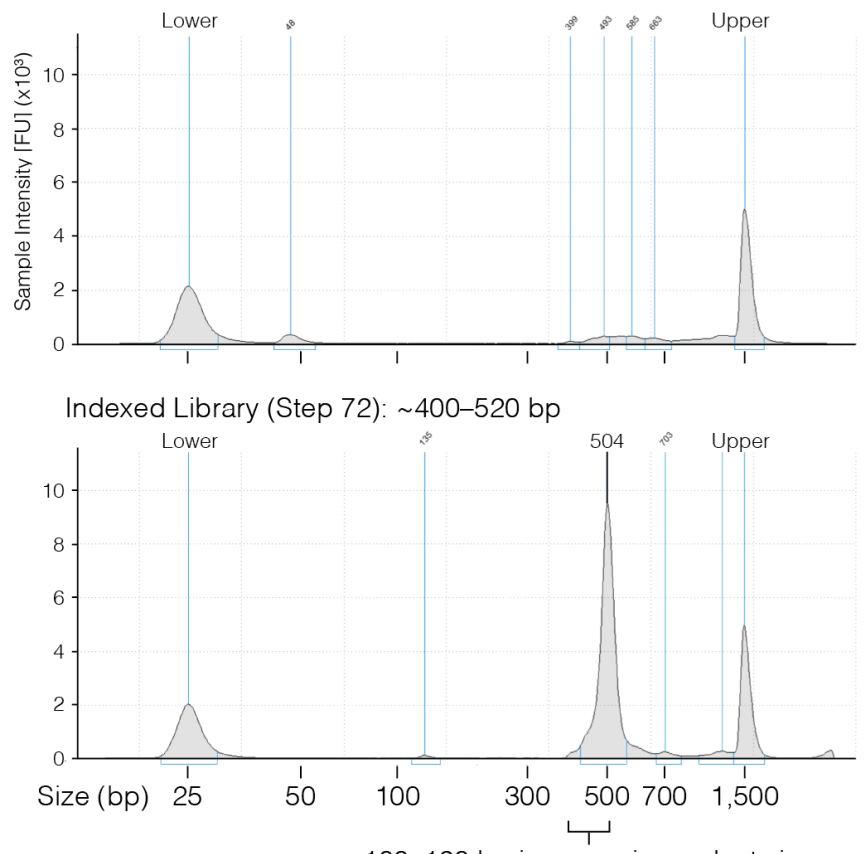

Figure 3. D1000 TapeStation traces of the barcoded amplicon, adaptor ligated fragments, and indexed amplicon show the increasing size of the DNA fragment with indexing. FU: Fluorescent units.

Table 4. Example results for screening of edited erythroid cells.

\begin{tabular}{cccc}
\hline & Wells Processed & Successfully Sequenced & Success Rate \\
\hline Locus 1 & 52 & 49 & $94.2 \%$ \\
Locus 2 & 244 & 217 & $88.9 \%$ \\
Locus 3 & 87 & 85 & $97.7 \%$ \\
Locus 4 & 59 & 53 & $89.8 \%$ \\
Locus 5 & 47 & 43 & $91.4 \%$ \\
Locus 6 & 74 & 72 & $97.2 \%$ \\
Locus 7 & 236 & 234 & $99.1 \%$ \\
\hline Total & 799 & 753 & $94.2 \%$ \\
\hline
\end{tabular}




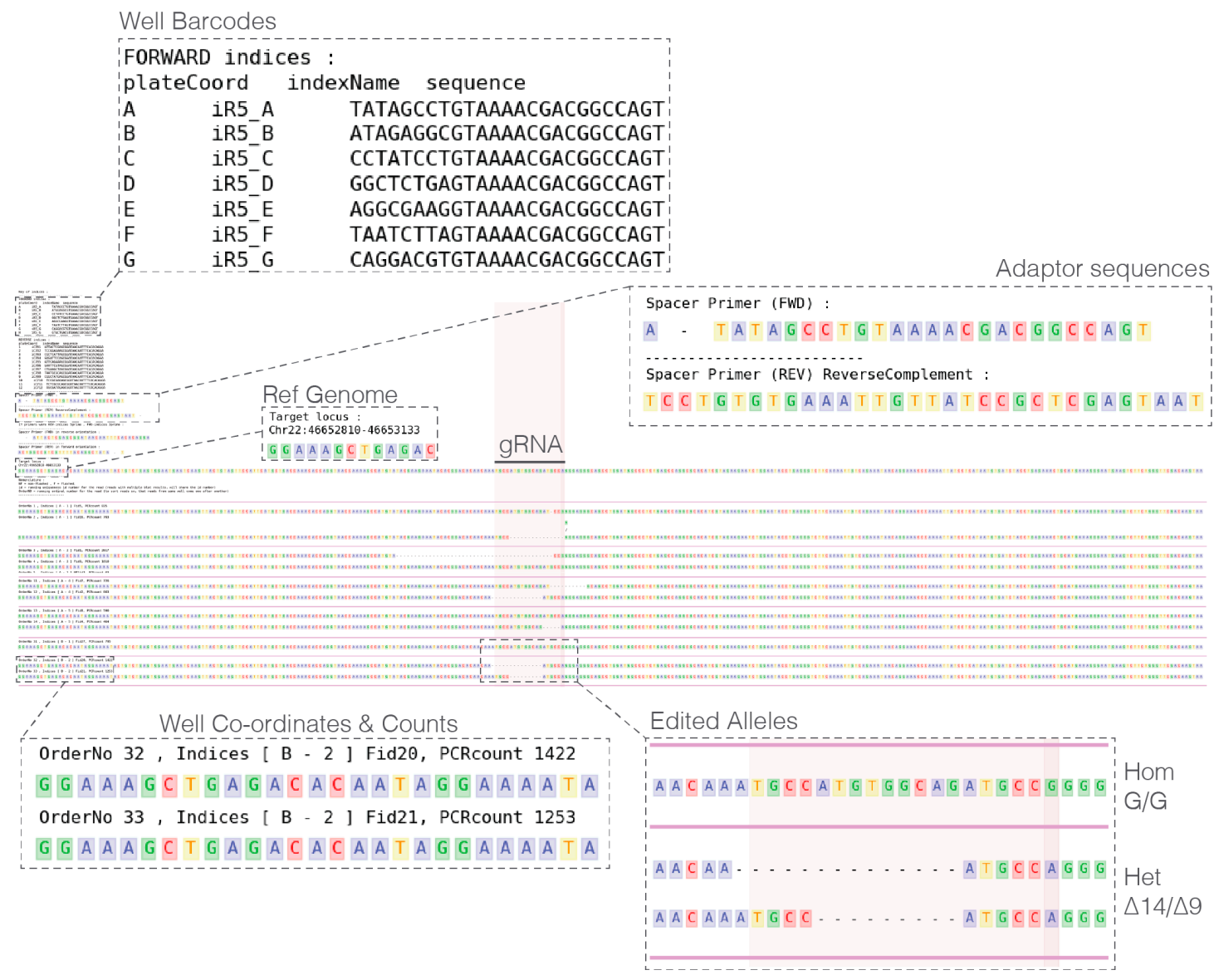

Figure 4. PlateScreen96 generates a PDF of a colored alignment of reads against the reference genome and reports input barcode and adaptor sequences and allows highlighting of key regions such as the guide RNA (gRNA). Reads are sorted per identified well and counts given, allowing users to assign genotypes for edited alleles. In this example, rs4508712 was edited from homozygous A/A to homozygous G/G.

\section{Reagents Setup}

\section{Cell Lysis Buffer}

Lysis Buffer (Table 5) can be made in advance by excluding proteinase $\mathrm{K}$ and stored at $4{ }^{\circ} \mathrm{C}$ for up to one month. Proteinase $\mathrm{K}$ should be added fresh on the day of use.

Table 5. Sufficient lysis buffer for one full 96-well plate.

\begin{tabular}{ccc}
\hline Reagent & Volume & Final Conc. \\
\hline 1 M Tris, pH 8.0 & $150 \mu \mathrm{L}$ & $50 \mathrm{mM}$ \\
0.5 M EDTA, pH 8.0 & $6 \mu \mathrm{L}$ & $1 \mathrm{mM}$ \\
50\% Tween 20 & $30 \mu \mathrm{L}$ & $0.5 \%$ \\
PCR Grade Water & $2.805 \mathrm{~mL}$ & - \\
Proteinase $\mathrm{K}(>0.6 \mathrm{U} / \mu \mathrm{L})$ & $9 \mu \mathrm{L}$ & $>0.0018 \mathrm{U} / \mu \mathrm{L}$ \\
\hline
\end{tabular}

Supplementary Materials: The following are available online at http:/ / www.mdpi.com/2409-9279/1/3/29/s1, Table S1: List of primers used to screen for editing.

Author Contributions: Conceptualization, L.N., J.M.T., M.C.S. and D.J.D.; Methodology, L.N., J.M.T. and M.C.S.; Software, J.M.T.; Formal Analysis, L.N., S.H., P.P.H., J.M.T.; Investigation, L.N., S.H. and P.P.H.; Resources, J.R.H.; 
Writing-Original Draft Preparation, D.J.D.; Writing-Review \& Editing, D.J.D and J.R.H.; Visualization, D.J.D.; Supervision, D.J.D.; Project Administration, J.R.H.; Funding Acquisition, J.R.H and T.W.C.

Funding: This research was funded by a Wellcome Trust strategic award 106130/Z/14/Z and Medical Research Council core funding MC_UU_12009/15.

Acknowledgments: We appreciate guidance and advice provided by Philip Hublitz from MRC Weatherall Institute of Molecular Medicine at the University of Oxford.

Conflicts of Interest: The authors declare no conflict of interest.

\section{References}

1. Bibikova, M.; Golic, M.; Golic, K.G.; Carroll, D. Targeted chromosomal cleavage and mutagenesis in Drosophila using zinc-finger nucleases. Genetics 2002, 161, 1169-1175. [PubMed]

2. Miller, J.C.; Tan, S.; Qiao, G.; Barlow, K.A.; Wang, J.; Xia, D.F.; Meng, X.; Paschon, D.E.; Leung, E.; Hinkley, S.J.; et al. A TALE nuclease architecture for efficient genome editing. Nat. Biotechnol. 2011, 29, 143-150. [CrossRef] [PubMed]

3. Cong, L.; Ran, F.A.; Cox, D.; Lin, S.; Barretto, R.; Habib, N.; Hsu, P.D.; Wu, X.; Jiang, W.; Marraffini, L.A.; et al. Multiplex Genome Engineering Using CRISPR/Cas Systems. Science 2013, 339, 819-823. [CrossRef] [PubMed]

4. Jinek, M.; Chylinski, K.; Fonfara, I.; Hauer, M.; Doudna, J.A.; Charpentier, E. A programmable dual-RNA-guided DNA endonuclease in adaptive bacterial immunity. Science 2012, 337, 816-822. [CrossRef] [PubMed]

5. Mali, P.; Yang, L.; Esvelt, K.M.; Aach, J.; Guell, M.; DiCarlo, J.E.; Norville, J.E.; Church, G.M. RNA-Guided Human Genome Engineering via Cas9. Science 2013, 339, 823-826. [CrossRef] [PubMed]

6. Gaudelli, N.M.; Komor, A.C.; Rees, H.A.; Packer, M.S.; Badran, A.H.; Bryson, D.I.; Liu, D.R. Programmable base editing of $A \bullet T$ to $G \bullet C$ in genomic DNA without DNA cleavage. Nature 2017, 551, 464-471. [CrossRef] [PubMed]

7. Miyaoka, Y.; Berman, J.R.; Cooper, S.B.; Mayerl, S.J.; Chan, A.H.; Zhang, B.; Karlin-Neumann, G.A.; Conklin, B.R. Systematic quantification of HDR and NHEJ reveals effects of locus, nuclease, and cell type on genome-editing. Sci. Rep. 2016, 6, 1-12. [CrossRef] [PubMed]

8. Kosicki, M.; Bradley, A. Repair of CRISPR-Cas9-induced double-stranded breaks leads to large deletions and complex rearrangements. Nature 2018. [CrossRef]

9. Wang, H.; Yang, H.; Shivalila, C.S.; Dawlaty, M.M.; Cheng, A.W.; Zhang, F.; Jaenisch, R. One-step generation of mice carrying mutations in multiple genes by CRISPR/cas-mediated genome engineering. Cell 2013, 153, 910-918. [CrossRef] [PubMed]

10. Shalem, O.; Sanjana, N.E.; Hartenian, E.; Shi, X.; Scott, D.A.; Mikkelson, T.; Heckl, D.; Ebert, B.L.; Root, D.E.; Doench, J.G.; et al. Genome-Scale CRISPR-Cas9 Knockout Screening in Human Cells. Science 2014, 343, 84-87. [CrossRef] [PubMed]

11. Koike-Yusa, H.; Li, Y.; Tan, E.P.; Velasco-Herrera, M.D.C.; Yusa, K. Genome-wide recessive genetic screening in mammalian cells with a lentiviral CRISPR-guide RNA library. Nat. Biotechnol. 2014, 32, 267-273. [CrossRef] [PubMed]

12. Wang, T.; Wei, J.J.; Sabatini, D.M.; Lander, E.S. Genetic Screens in Human Cells Using the CRISPR-Cas9 System. Science 2014, 343, 80-84. [CrossRef] [PubMed]

13. Zhou, Y.; Zhu, S.; Cai, C.; Yuan, P.; Li, C.; Huang, Y.; Wei, W. High-throughput screening of a CRISPR/Cas9 library for functional genomics in human cells. Nature 2014, 509, 487-491. [CrossRef] [PubMed]

14. Chen, F.; Pruett-Miller, S.M.; Huang, Y.; Gjoka, M.; Duda, K.; Taunton, J.; Collingwood, T.N.; Frodin, M.; Davis, G.D. High-frequency genome editing using ssDNA oligonucleotides with zinc-finger nucleases. Nat. Methods 2011, 8, 753-757. [CrossRef] [PubMed]

15. Soldner, F.; Stelzer, Y.; Shivalila, C.S.; Abraham, B.J.; Latourelle, J.C.; Barrasa, M.I.; Goldmann, J.; Myers, R.H.; Young, R.A.; Jaenisch, R. Parkinson-associated risk variant in distal enhancer of $\alpha$-synuclein modulates target gene expression. Nature 2016, 533, 95-99. [CrossRef] [PubMed]

16. Yu, C.; Liu, Y.; Ma, T.; Liu, K.; Xu, S.; Zhang, Y.; Liu, H.; La Russa, M.; Xie, M.; Ding, S.; et al. Small molecules enhance CRISPR genome editing in pluripotent stem cells. Cell Stem Cell 2015, 16, 142-147. [CrossRef] [PubMed] 
17. Yu, C.; Zhang, Y.; Yao, S.; Wei, Y. A PCR Based Protocol for Detecting Indel Mutations Induced by TALENs and CRISPR/Cas9 in Zebrafish. PLoS ONE 2014, 9, e98282. [CrossRef] [PubMed]

18. Ramlee, M.K.; Yan, T.; Cheung, A.M.S.; Chuah, C.T.H.; Li, S. High-throughput genotyping of CRISPR/Cas9-mediated mutants using fluorescent PCR-capillary gel electrophoresis. Sci. Rep. 2015, 5, 1-13. [CrossRef] [PubMed]

19. Samarut, É.; Lissouba, A.; Drapeau, P. A simplified method for identifying early CRISPR-induced indels in zebrafish embryos using High Resolution Melting analysis. BMC Genom. 2016, 17, 1-6. [CrossRef] [PubMed]

20. Yang, L.; Yang, J.L.; Byrne, S.; Pan, J.; Church, G.M. CRISPR/Cas9-Directed Genome Editing of Cultured Cells. Curr. Protocols Mol. Biol. 2014, 107, 31.1.1-31.1.17.

21. Schmid-Burgk, J.L.; Schmidt, T.; Gaidt, M.M.; Pelka, K.; Latz, E.; Ebert, T.S.; Hornung, V. OutKnocker: A web tool for rapid and simple genotyping of designer nuclease edited cell lines OutKnocker: A web tool for rapid and simple genotyping of designer nuclease edited cell lines. Genome Res. 2014, 1719-1723. [CrossRef] [PubMed]

22. Ran, F.A.; Hsu, P.D.; Wright, J.; Agarwala, V.; Scott, D.A.; Zhang, F. Genome engineering using the CRISPR-Cas9 system. Nat. Protoc. 2013, 8, 2281-2308. [CrossRef] [PubMed]

23. Moir-Meyer, G.; Cheong, P.L.; Olijnik, A.; Brown, J.; Knight, S.; King, A.; Kurita, R.; Nakamura, Y.; Gibbons, R.; Higgs, D.; et al. Robust CRISPR/Cas9 Genome Editing of the HUDEP-2 Erythroid Precursor Line Using Plasmids and Single-Stranded Oligonucleotide Donors. Methods Protoc. 2018, 1, 28. [CrossRef]

24. Telenius, J.M. plateScreen96 (GitHub). Available online: github.com/Hughes-Genome-Group/ plateScreen96/releases (accessed on 26 July 2018).

25. Telenius, J.M. plate96 Guide. plateSreen96-CRISPR short indel genotype analyser. Available online: http:/ / sara.molbiol.ox.ac.uk/public/telenius/plate96 (accessed on 26 July 2018).

26. Integrated DNA Technologies (IDT). PrimerQuest Tool. Available online: www.idtdna.com/Primerquest (accessed on 26 July 2018).

27. Kent, W.J. BLAT-The BLAST-Like Alignment Tool. Genome Res. 2002, 12, 656-664. [CrossRef] [PubMed]

(C) 2018 by the authors. Licensee MDPI, Basel, Switzerland. This article is an open access article distributed under the terms and conditions of the Creative Commons Attribution (CC BY) license (http:/ / creativecommons.org/licenses/by/4.0/). 